Difference Resonances in a controlled van der Pol-Duffing oscillator involving time delay

This paper was published in the journal

Chaos, Solitions \& Fractals, vol.42, no.2, pp.975-980, Oct 2009

\author{
J.C. Ji, N. Zhang, Wei Gao \\ Faculty of Engineering, University of Technology, Sydney \\ PO Box 123, Broadway, NSW 2007, Australia
}

The current address for correspondence:

Dr. J. C. Ji

Faculty of Engineering

Level 20, Building 1

University of Technology, Sydney

PO Box 123, Broadway, NSW 2007

Australia

Email: jin.ji@uts.edu.au

Phone: ++61 295142412

Total number of pages including figures: $10+3=13$

Total number of figures: 3 


\title{
Difference Resonances in a controlled van der Pol-Duffing oscillator involving time delay
}

\author{
J.C. Ji, N. Zhang, Wei Gao \\ Faculty of Engineering, University of Technology, Sydney \\ PO Box 123, Broadway, NSW 2007, Australia
}

\begin{abstract}
A non-resonant interaction of two Hopf bifurcations may appear after the trivial solution of a controlled van der Pol-Duffing oscillator without external excitation loses its stability, when two critical time delays corresponding to two Hopf bifurcations have the same value. In the vicinity of the non-resonant Hopf bifurcations, the presence of a periodic excitation in the controlled oscillator can induce difference resonances in the forced response, when the forcing frequency and the frequencies of the two Hopf bifurcations satisfy certain relationships. It is found that the frequency response curves of the controlled system under difference resonances are an isolated closed curve. The difference resonance response may admit two stable motions on a three-dimensional torus consisting of three frequencies. Illustrative examples are given to show the quasi-periodic motions.
\end{abstract}

\section{Introduction}

Time delayed feedback control has been applied to suppress nonlinear vibrations and stabilise bifurcations of nonlinear systems with parametric (or external) excitations [1-5]. In addition to the selection of control gains, effective vibration control requires an optimal selection of the appropriate values of time delays to achieve satisfactory control performance. Usually the time delays are selected to be well separated from the critical values at which the trivial solution of the corresponding autonomous system loses its stability. It is now accepted that an introduction of time delays in a nonlinear system can 
induce certain dynamic behaviour such as instability, bifurcations and chaotic motions [613], which may be undesirable from the perspective of vibration control.

For a nonlinear system having external excitation, the presence of time delays may lead to distinct behaviour that cannot be observed in the same nonlinear system without time delay. An important phenomenon is the interaction of the periodic external excitation and the stable periodic solutions that result from Hopf bifurcations of the corresponding autonomous system. In particular, for a nonlinear system losing the stability of its trivial solution through a single Hopf bifurcation, the interaction may lead to primary resonances, sub-harmonic and super-harmonic resonances in the forced response when the frequency of the Hopf bifurcation and the forcing frequency satisfy a certain relationship. More interestingly, for a nonlinear system losing the stability of its trivial solution via two Hopf bifurcations, the interaction can further result in additive resonances and difference resonances, when the forcing frequency and the frequencies of the two Hopf bifurcations satisfy certain relationships. The main purpose of the present paper is to study difference resonance response of a controlled van der Pol-Duffing oscillator subjected to a periodic excitation, which results from an interaction of the external excitation and two periodic solutions bifurcated from the trivial solution of the corresponding autonomous system at non-resonant Hopf bifurcations.

An externally forced van der Pol-Duffing oscillator under a linear-plus-nonlinear feedback control considered in the present paper is of the form

$$
\begin{gathered}
\ddot{x}-\left(\mu-\beta x^{2}\right) \dot{x}+\omega^{2} x+\alpha x^{3}=e_{0} \cos \left(\Omega_{0} t\right)+p x(t-\tau)+q \dot{x}(t-\tau)+k_{1} x^{3}(t-\tau) \\
+k_{2} \dot{x}^{3}(t-\tau)+k_{3} \dot{x}(t-\tau) x^{2}(t-\tau)+k_{4} \dot{x}^{2}(t-\tau) x(t-\tau)
\end{gathered}
$$

where $x$ is the displacement, an overdot indicates the differentiation with respect to time $t$, $\omega$ is the natural frequency, $\alpha$ is the coefficient of the nonlinear term, $\mu$ and $\beta$ are the linear and nonlinear damping coefficients with $\mu>0, \beta>0, e_{0}$ and $\Omega_{0}$ represent the amplitude and frequency of the external excitation, $p, q$ and $k_{i}(i=1,2,3,4)$ are linear and 
weakly nonlinear feedback gains, respectively, and $\tau$ denotes the time delay occurring in the feedback path. Only one time delay is considered here for simplicity.

With the increase of the value of time delay, the trivial solution of the corresponding autonomous system (obtained by letting $e_{0}=0$ in equation (1)) was found to lose its stability via a subcritical or supercritical Hopf bifurcation and regain its stability via a reverse subcritical or supercritical Hopf bifurcation [14]. An intersection of two nonresonant Hopf bifurcations may occur on the stability boundary of the trivial solution. The point of the intersection is usually associated with the bifurcation of co-dimension two and may be a source of complicated behaviour in the context of ordinary differential equations $[15,16]$. It was found that the autonomous system may admit the trivial solution, two periodic solutions and a quasi-periodic solution on a two-dimensional (2D) torus [17].

In the neighbourhood of non-resonant Hopf bifurcations, the presence of an external periodic excitation can further induce rich dynamic behaviour of the controlled oscillator (1), which includes primary resonances, sub-harmonic and super-harmonic resonances, additive and difference resonances [18]. These phenomena are hard to identify from the original system (1), as they do not relate to the so-called natural frequency $\omega$ (nor to its multiples). Understanding of the distinct observable behaviour in nonlinear systems involving time delays remains limited. The non-resonant response and two types of primary resonances of the controlled oscillator were studied in reference [18] and three types of additive resonances were discussed in reference [19]. Difference resonance response has not yet attracted much attention even in the context of non-linear oscillations to the authors' best knowledge. Two types of difference resonance responses will be discussed in the present paper.

The present paper is organised into five sections. Section 2 presents four first-order ordinary differential equations on the centre manifold. Section 3 lists types of resonances 
that can occur in the forced response. Two types of difference resonance responses are studied in Section 4. Conclusion is given in Section 5.

\section{Reduction of the centre manifold}

The dynamic behaviour of the solutions of equation (1) in the vicinity of non-resonant Hopf bifurcations can be interpreted by the solutions and their stability of four ordinary differential equations on the centre manifolds, which are expressed in component form as

$$
\begin{aligned}
& \dot{z}_{1}=l_{11} z_{1}+\left(\delta_{1}+l_{12}\right) z_{2}+l_{13} z_{3}+l_{14} z_{4}+f_{10}\left(z_{1}, z_{2}, z_{3}, z_{4}\right)+e_{10} \cos (\Omega t), \\
& \dot{z}_{2}=\left(-\delta_{1}+l_{21}\right) z_{1}+l_{22} z_{2}+l_{23} z_{3}+l_{24} z_{4}+f_{20}\left(z_{1}, z_{2}, z_{3}, z_{4}\right)+e_{20} \cos (\Omega t), \\
& \dot{z}_{3}=l_{31} z_{1}+l_{32} z_{2}+l_{33} z_{3}+\left(\delta_{2}+l_{34}\right) z_{4}+f_{30}\left(z_{1}, z_{2}, z_{3}, z_{4}\right)+e_{30} \cos (\Omega t), \\
& \dot{z}_{4}=l_{41} z_{1}+l_{42} z_{2}+\left(-\delta_{2}+l_{43}\right) z_{3}+l_{44} z_{4}+f_{40}\left(z_{1}, z_{2}, z_{3}, z_{4}\right)+e_{40} \cos (\Omega t),
\end{aligned}
$$

where $\delta_{1}$ and $\delta_{2}$ are the normalized frequencies of Hopf bifurcations. $f_{i 0}\left(z_{1}, z_{2}, z_{3}, z_{4}\right)$ $(i=1,2,3,4$. $)$ are polynomial functions of order three in terms of $z_{1}^{k} z_{2}^{l} z_{3}^{m} z_{4}^{n}$ with $k+l+m+n=3$ and $k, l, m, n=0,1,2,3$. More details on the coefficients and relevant algebraic manipulations can be found in the reference [17].

\section{Types of resonances}

When two natural frequencies (or their combinations) and the forcing frequency satisfy certain relationships, the forced response of the system (2) may exhibit non-resonant response and a number of resonant responses. In particular, two types of primary resonances may occur when $\delta_{1} \cong \Omega$ or $\delta_{2} \cong \Omega$. Two types of sub-harmonic resonances happen when $3 \delta_{1} \cong \Omega$ or $3 \delta_{2} \cong \Omega$, and two types of super-harmonic resonances take place when $\delta_{1} \cong 3 \Omega$ or $\delta_{2} \cong 3 \Omega$. For $\delta_{1}>\delta_{2}$, a number of combinational resonances of additive and difference types may appear in the forced response. Specifically, additive resonances may appear when either $\delta_{1}+\delta_{2} \cong 2 \Omega$, or $2 \delta_{1}+\delta_{2} \cong \Omega$, or $\delta_{1}+2 \delta_{2} \cong \Omega$. Difference resonances may occur when either $\delta_{1}-\delta_{2} \cong 2 \Omega$, or $\delta_{1}-2 \delta_{2} \cong 2 \Omega$, or 
$2 \delta_{1}-\delta_{2} \cong \Omega$; or $2 \delta_{2}-\delta_{1} \cong \Omega$. Accordingly, a total of 13 types of nonlinear responses may take place in the forced nonlinear response. Thus in studying the forced response of the nonlinear system (2), six cases need to be distinguished based on six categories of resonances discussed above, which are (a) primary resonances at either frequency of two Hopf bifurcations; (b) sub-harmonic resonances at either of two Hopf bifurcation frequencies; (c) super-harmonic resonances at either of two Hopf bifurcation frequencies; (d) additive resonances; (e) difference resonances; and (f) non-resonant response when the forcing frequency $\Omega$ is well separated from the above-mentioned resonances.

\section{Difference resonances}

By using the method of multiple scales [20], the first-order solutions to equation (2) can be written in a general form as

$$
\begin{aligned}
& z_{11}=r_{1} \cos \left(\delta_{1} T_{0}+\phi_{1}\right)+A_{1} \cos \left(\Omega T_{0}\right)+A_{2} \sin \left(\Omega T_{0}\right), \\
& z_{21}=-r_{1} \sin \left(\delta_{1} T_{0}+\phi_{1}\right)+B_{1} \cos \left(\Omega T_{0}\right)+B_{2} \sin \left(\Omega T_{0}\right), \\
& z_{31}=r_{2} \cos \left(\delta_{2} T_{0}+\phi_{2}\right)+A_{3} \cos \left(\Omega T_{0}\right)+A_{4} \sin \left(\Omega T_{0}\right), \\
& z_{41}=-r_{2} \sin \left(\delta_{2} T_{0}+\phi_{2}\right)+B_{3} \cos \left(\Omega T_{0}\right)+B_{4} \sin \left(\Omega T_{0}\right),
\end{aligned}
$$

where $r_{1}, r_{2}, \phi_{1}, \phi_{2}$ represent the amplitudes and phases of the free-oscillation terms, and the coefficients $A$ and $B$ 's denote the amplitudes of the particular solutions.

As discussed in Section 3, four types of difference resonances may appear in the forced response. The present paper will study two cases of difference resonances, namely $\delta_{1}-\delta_{2} \cong 2 \Omega, 2 \delta_{1}-\delta_{2} \cong \Omega$. The other cases can be studied in a similar manner. To account for the nearness of the forcing frequency to the combination of two natural frequencies, two detuning parameters, $\sigma_{1}$ and $\sigma_{2}$, are introduced in terms of $2 \Omega=\delta_{1}-\delta_{2}+\varepsilon \sigma_{1}, \quad \Omega=2 \delta_{1}-\delta_{2}+\varepsilon \sigma_{2}$. These two types of difference resonances will be referred to here as Cases I and II. 
Case I: $2 \Omega=\delta_{1}-\delta_{2}+\varepsilon \sigma_{1}$

The averaged equations that determine the amplitudes and phases of the response of difference resonance Case I are given by

$$
\begin{aligned}
& \dot{r}_{1}=-\mu_{1} r_{1}+s_{11} r_{1}^{3}+s_{12} r_{1} r_{2}^{2}+s_{13} r_{2} \cos \left(\gamma_{11}+\gamma_{12}\right)-s_{33} r_{2} \sin \left(\gamma_{11}+\gamma_{12}\right), \\
& r_{1} \dot{\gamma}_{11}=\left(\frac{1}{2} \sigma_{1}-\rho_{1}\right) r_{1}-s_{31} r_{1}^{3}-s_{32} r_{1} r_{2}^{2}-s_{13} r_{2} \sin \left(\gamma_{11}+\gamma_{12}\right)-s_{33} r_{2} \cos \left(\gamma_{11}+\gamma_{12}\right), \\
& \dot{r}_{2}=-\mu_{2} r_{2}+s_{21} r_{1}^{2} r_{2}+s_{22} r_{2}^{3}+s_{23} r_{1} \cos \left(\gamma_{11}+\gamma_{12}\right)+s_{43} r_{1} \sin \left(\gamma_{11}+\gamma_{12}\right), \\
& r_{2} \dot{\gamma}_{12}=\left(\frac{1}{2} \sigma_{1}+\rho_{2}\right) r_{2}+s_{41} r_{1}^{2} r_{2}+s_{42} r_{2}^{3}-s_{23} r_{1} \sin \left(\gamma_{11}+\gamma_{12}\right)+s_{43} r_{1} \cos \left(\gamma_{11}+\gamma_{12}\right),
\end{aligned}
$$

where $\gamma_{11}=\frac{1}{2} \sigma_{1} T_{0}-\phi_{1}, \gamma_{12}=\frac{1}{2} \sigma_{1} T_{0}+\phi_{2}$, the coefficients are not reproduced here.

The steady-state difference resonance response can be studied by finding the solutions to the four algebraic equations which can be obtained by letting $\dot{r}_{1}=\dot{r}_{2}=0$ and $\dot{\gamma}_{11}=\dot{\gamma}_{12}=0$ in equation (4). The frequency response equations are given by

$$
\begin{aligned}
& \left(-\mu_{1}+s_{11} r_{1}^{2}+s_{12} r_{2}^{2}\right)^{2} r_{1}^{2}+\left(\frac{1}{2} \sigma_{1}-\rho_{1}-s_{31} r_{1}^{2}-s_{32} r_{2}^{2}\right)^{2} r_{1}^{2}=\left(s_{13}^{2}+s_{33}^{2}\right) r_{2}^{2}, \\
& \left(-\mu_{2}+s_{21} r_{1}^{2}+s_{22} r_{2}^{2}\right)^{2} r_{2}^{2}+\left(\frac{1}{2} \sigma_{1}+\rho_{2}+s_{41} r_{1}^{2}+s_{42} r_{2}^{2}\right)^{2} r_{2}^{2}=\left(s_{23}^{2}+s_{43}^{2}\right) r_{1}^{2} .
\end{aligned}
$$

Real positive solutions of equation (5) lead to the frequency-response curves. The stability of the solutions can be examined by computing the eigenvalues of the coefficient matrix of characteristic equations which are derived from equation (4) in terms of small disturbances to the steady state solutions.

Case II: $\Omega=2 \delta_{1}-\delta_{2}+\varepsilon \sigma_{2}$

The averaged equations for difference resonance Case 2 are given by

$$
\begin{aligned}
& \dot{r}_{1}=-\mu_{1} r_{1}+s_{11} r_{1}^{3}+s_{12} r_{1} r_{2}^{2}+s_{14} r_{1} r_{2} \cos \left(2 \gamma_{21}+\gamma_{22}\right)-s_{34} r_{1} r_{2} \sin \left(2 \gamma_{21}+\gamma_{22}\right), \\
& r_{1} \dot{\gamma}_{21}=\left(\frac{1}{3} \sigma_{2}-\rho_{1}\right) r_{1}-s_{31} r_{1}^{3}-s_{32} r_{1} r_{2}^{2}-s_{14} r_{1} r_{2} \sin \left(2 \gamma_{21}+\gamma_{22}\right)-s_{34} r_{1} r_{2} \cos \left(2 \gamma_{21}+\gamma_{22}\right), \\
& \dot{r}_{2}=-\mu_{2} r_{2}+s_{21} r_{1}^{2} r_{2}+s_{22} r_{2}^{3}+s_{24} r_{1}^{2} \cos s\left(2 \gamma_{21}+\gamma_{22}\right)+s_{44} r_{1}^{2} \sin \left(2 \gamma_{21}+\gamma_{22}\right), \\
& r_{2} \dot{\gamma}_{22}=\left(\frac{1}{3} \sigma_{2}+\rho_{2}\right) r_{2}+s_{41} r_{1}^{2} r_{2}+s_{42} r_{2}^{3}-s_{24} r_{1}^{2} \sin \left(2 \gamma_{21}+\lambda_{22}\right)+s_{44} r_{1}^{2} \cos \left(2 \gamma_{21}+\gamma_{22}\right),
\end{aligned}
$$


where $\gamma_{21}=\frac{1}{3} \sigma_{2} T_{0}-\phi_{1}, \gamma_{22}=\frac{1}{3} \sigma_{2} T_{0}+\phi_{2}$, the coefficients are not produced here.

The frequency response curves can be determined by numerically solving the equations:

$$
\begin{aligned}
& \left(-\mu_{1}+s_{11} r_{1}^{2}+s_{12} r_{2}^{2}\right)^{2} r_{1}^{2}+\left(\frac{1}{3} \sigma_{2}-\rho_{1}-s_{31} r_{1}^{2}-s_{32} r_{2}^{2}\right)^{2} r_{1}^{2}=\left(s_{14}^{2}+s_{34}^{2}\right) r_{1}^{2} r_{2}^{2}, \\
& \left(-\mu_{2}+s_{21} r_{1}^{2}+s_{22} r_{2}^{2}\right)^{2} r_{2}^{2}+\left(\frac{1}{3} \sigma_{2}+\rho_{2}+s_{41} r_{1}^{2}+s_{42} r_{2}^{2}\right)^{2} r_{2}^{2}=\left(s_{24}^{2}+s_{44}^{2}\right) r_{1}^{4} .
\end{aligned}
$$

Equation (7) admits two types of solutions which are referred to here as the single-mode solutions with $r_{1}=0$ and $r_{2} \neq 0$, and the mixed-mode solutions with $r_{1} \neq 0$ and $r_{2} \neq 0$.

As illustrative examples, consider a specific system with parameters $\mu=0.1, \omega=1.0$, $p=-0.4, \quad q=-0.40219, \quad \alpha=0.4, \quad \beta=0.5, \quad k_{1}=0.2, \quad k_{4}=0.5, \quad k_{2}=k_{3}=0.0$. The frequencies of non-resonant Hopf bifurcations were found to be $\delta_{+}=1.28038$ and $\delta_{-}=0.71582$. Difference resonances may appear in the neighbourhood of $\Omega_{0}=0.28228$ for Case I, and $\Omega_{0}=1.84494$ for Case II.

When $\alpha_{1}=-0.01, \alpha_{2}=0.002, e_{0}=0.14, \Omega_{0}=0.28228$, the frequency-response curves for difference resonance Case I are shown in Figure 1. Stable solutions are represented by circles and unstable solutions by crosses. The frequency response curve is an isolated closed curve in Figures 1(a) and (b). The difference resonance exists only in a small interval of the external detuning $\sigma_{1}$ and does not exist outside this closed curve. The closed curve consists of two branches. The upper branch of $r_{1}$ is stable with the four eigenvalues having negative real part. The lower branch of $r_{1}$ is unstable with one eigenvalue having positive real part. On the contrary, the upper branch of $r_{2}$ is unstable and the lower branch of $r_{2}$ is stable. As $\sigma_{1}$ increases from a small value, the amplitude $r_{1}$ of the free-oscillation term with natural frequency $\delta_{1}$ grows, whereas the amplitude $r_{2}$ of the free-oscillation term having natural frequency $\delta_{2}$ decreases. The amplitude $r_{1}$ is much higher than amplitude $r_{2}$, which means the motion corresponding to $\delta_{1}$ dominates the response while the motion relating to $\delta_{2}$ is smaller in amplitude but not negligible. Stable 
non-trivial solutions indicate that the forced response under difference resonances comprises both the free-oscillation terms and forced terms as given in equation (2). Because two natural frequencies $\delta_{1}$ and $\delta_{2}$ are incommensurable, the system response under difference resonance Case I exhibits quasi-periodic motions.

Figure 2 shows frequency-response curves for difference resonance Case II under $\alpha_{1}=0.001, \alpha_{2}=-0.0053, e_{0}=0.16, \Omega_{0}=1.845$. The frequency response curve is an isolated closed curve. The upper branch of the curve comprises two parts; stable part and unstable part. The lower branch is stable. There exists a region of bi-stability in the closed curve. In the interval $\sigma_{2} \in[0.015815,0.01695]$, two stable solutions coexist for the amplitudes $r_{1}$ and $r_{2}$. Amplitude $r_{1}$ is much higher than $r_{2}$, which means the motion corresponding to $\delta_{1}$ dominates the response. As the detuning $\sigma_{2}$ increases along the upper branch, both amplitudes $r_{1}$ and $r_{2}$ increases till $\sigma_{2}=0.01695$, where the stable mixed-mode solution loses its stability with one eigenvalue being zero. The other meeting point of stable and unstable branches is at $\sigma_{2}=0.01938$, where a saddle-node bifurcation occurs with one eigenvalues being zero.

Two stable solutions coexist in the region $\sigma_{2} \in[0.015815,0.01695]$, where two quasiperiodic solutions appear when doing numerical simulations to equation (1) under different sets of initial conditions. Figure 3 shows the phase portraits of two quasi-periodic motions at $\Omega_{0}=1.86094$ under different sets of initial conditions. The large-amplitude quasi-periodic motion shown in Figure 3(a) corresponds to a mixed-mode stable solution in the upper branch shown in Figure 2, while the small-amplitude quasi-periodic motion shown in Figure 3(b) relates to a mixed-mode stable solution in the lower branch. The quasi-periodic motions shown in Figure 3 contain three individual harmonic components: $\delta_{1}, \delta_{2}$ and $\Omega_{0}$. These three frequencies satisfy the difference resonance condition for Case II. The numerical results are in good agreement with the analytical predictions. 


\section{Conclusion}

Two types of difference resonances have been studied using the method of multiple scales. Illustrative examples have been given to demonstrate the frequency-response curves and quasi-periodic motions. It was shown that the difference resonance response of the forced oscillator exhibits quasi-periodic motions on a 3D torus. The quasi-periodic motion consists of three components having the frequencies of two Hopf bifurcations and the frequency of the excitation, which satisfy the difference resonance conditions. The difference resonance response may lose its stability via a saddle-node bifurcation. Two stable solutions exist in the forced response of the system under difference resonances. The co-existence of two quasi-periodic motions has been validated by numerical integration using different sets of initial conditions.

\section{Acknowledgment}

This research is partially supported by the Centre for Intelligent Mechatronic Systems (IMS centre) at UTS.

\section{References}

[1] Maccari, A., 2008 Arbitrary amplitude periodic solutions for parametrically excited systems with time delay, Nonlinear Dynamics, vol.51, pp.111-126.

[2] Maccari, A., 2007 Delayed feedback control for a parametrically excited van der Pol oscillator, Physica Scripta, vol.76, pp.526-532.

[3] Chatterjee, S., 2007 Time-delayed feedback control of friction-induced instability, International Journal of Non-Linear Mechanics, vol.42, pp.1127-1143.

[4] Maccari A, 2006 Vibration control for parametrically excited Lienard systems, International Journal of Non-Linear Mechanics, vol.41, pp.146-155.

[5] Ji, JC, Leung, AYT., 2002 Bifurcation control of a parametrically excited Duffing system, Nonlinear Dynamics, vol.27, pp.411-417.

[6] Niu, B., Wei, JJ., 2008 Stability and bifurcation analysis in an amplitude equation with delayed feedback, Chaos, Solitons \& Fractals, vol.37, pp.1362-1371. 
[7] Sah, S., Belhaq, M., 2008 Effect of vertical high-frequency parametric excitation on self-excited motion in a delayed van der Pol oscillator, Chaos, Solitons \& Fractals, vol.37, pp.1489-1496.

[8] Belhaq, M, Sah, SM., 2008 Fast parametrically excited van der Pol oscillator with time delay state feedback, International Journal of Non-Linear Mechanics, vol.43, pp.124130.

[9] EI-Bassioouny, AF, 2006 Stability and oscillation of two coupled Duffing equations with time delay state feedback, Physica Scripta, vol.74, pp.726-735.

[10] Lazarevic, MP., 2006 Finite time stability analysis of PD alpha fractional control of robotic time-delay systems, Mechanics Research Communications, vol.33, pp.269279.

[11] Xu, J., Yu, P., 2004, Delay-induced bifurcation in an nonautonomous system with delayed velocity feedbacks. International Journal of Bifurcation and Chaos, vol.14, pp.1777-1798.

[12] Maccari, A., 2001 The response of a parametrically excited van der Pol oscillator to a time delay state feedback, Nonlinear Dynamics, vol.26, pp105-119.

[13] Campbell, S.A., Belair, J., 1999 Resonant co-dimension two bifurcation in the harmonic oscillator with delayed forcing, Canadian Applied Mathematics Quarterly, vol.7, pp.218-238.

[14] Ji, J.C., Hansen, C.H., 2006 Stability and dynamics of a controlled van der PolDuffing oscillator, Chaos, Solitons and Fractals, vol.28(2), pp.555-570.

[15] Guckenheimer, J., Holmes, P., Nonlinear Oscillations, Dynamical Systems, and Bifurcations of Vector Fields, Springer-verlag, New York, 1983.

[16] Itovich, G.R., Moiola, J.L, 2005 Double Hopf bifurcation analysis using frequency domain methods, Nonlinear Dynamics, vol.39, pp.235-258.

[17] Ji, J.C., 2006 Nonresonant Hopf bifurcations of a controlled van der Pol-Duffing oscillator, Journal of Sound and Vibration, vol.297(1-2), pp.183-199.

[18] Ji, J.C., Zhang, N., 2008 Nonlinear response of a froced van der Pol-Duffing oscillator at non-resonant bifurcations of codimension two, Chaos, Solitons \& Fractals, In press (doi:10.1016/j.chaos.2008.06.008).

[19] Ji, J.C., Zhang, N., 2008 Additive resonances of a controlled van der Pol-Duffing oscillator, Journal of Sound and Vibration, vol.315, pp.22-33.

[20] Nayfeh, A.H., Mook, D.T., Nonlinear Oscillations, Wiley, New York, 1979. 


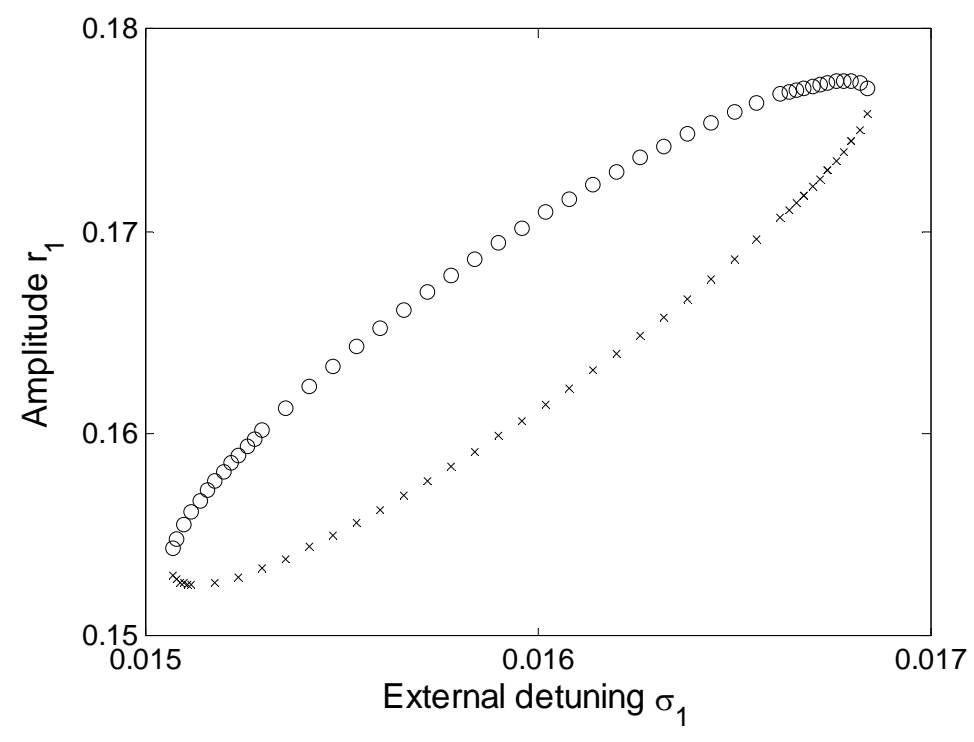

(a) the variation of amplitude $r_{1}$ with external detuning

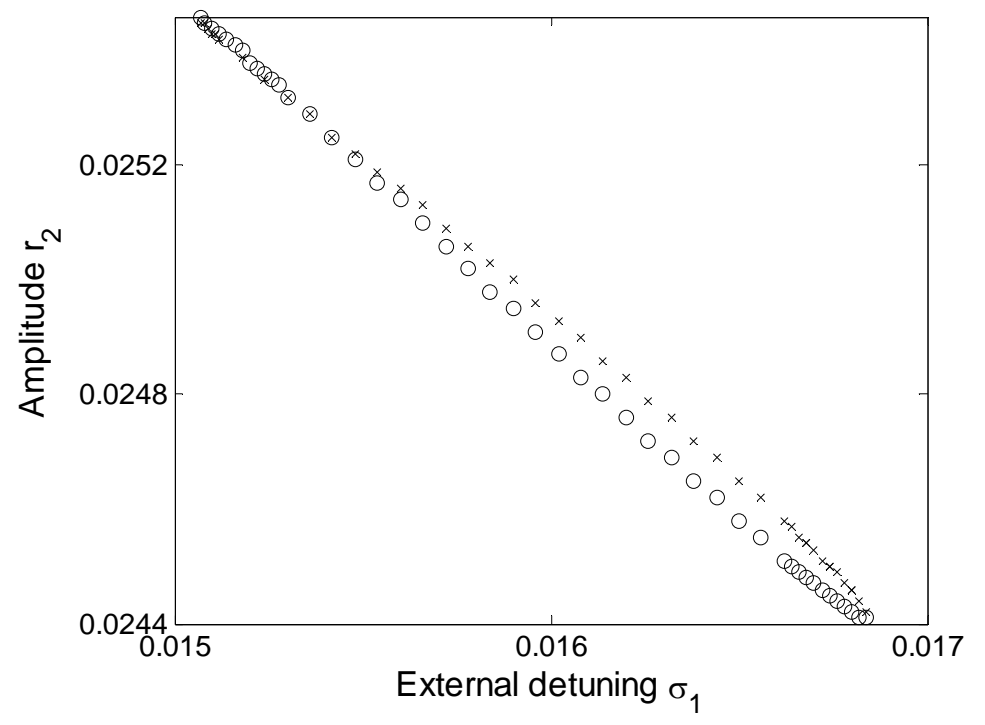

(b) the variation of amplitude $r_{2}$ with external detuning $\sigma_{1}$.

Figure 1. The frequency-response curves for difference resonance Case I for $\alpha_{1}=-0.01$, $\alpha_{2}=0.002, e_{0}=0.14, \Omega_{0}=0.28228$; (a) the variation of amplitude $r_{1}$ with external detuning $\sigma_{1}$, (b) the variation of amplitude $r_{2}$ with external detuning $\sigma_{1}$. Stable solutions are denoted by circles while unstable solutions by crosses. 


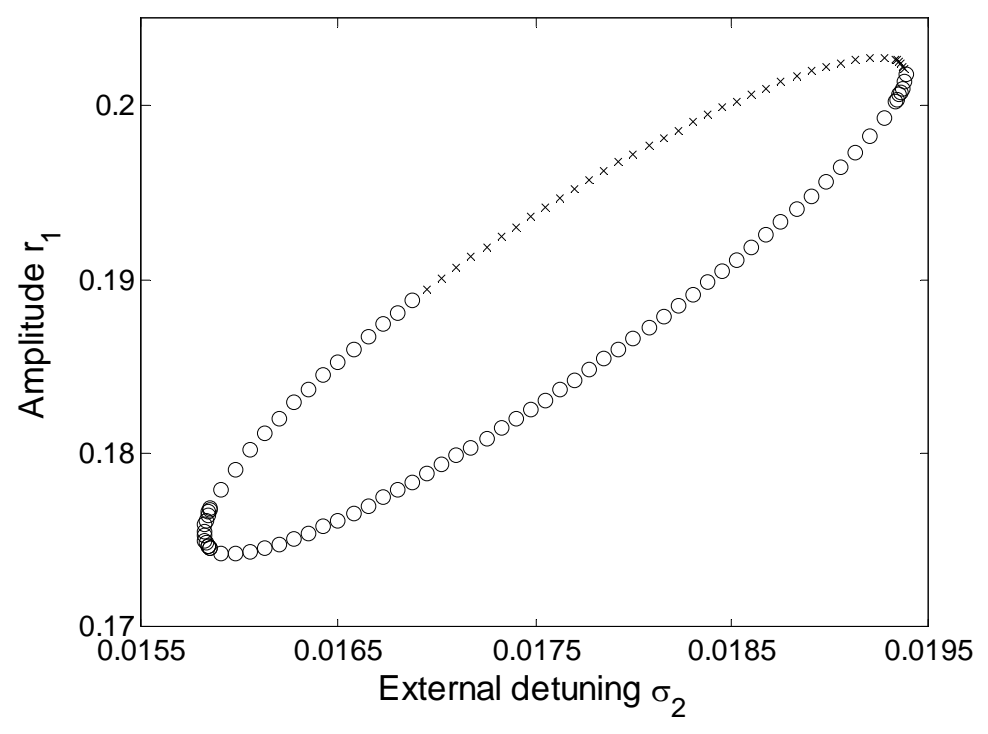

(a) the variation of amplitude $r_{1}$ with external detuning $\sigma_{2}$.

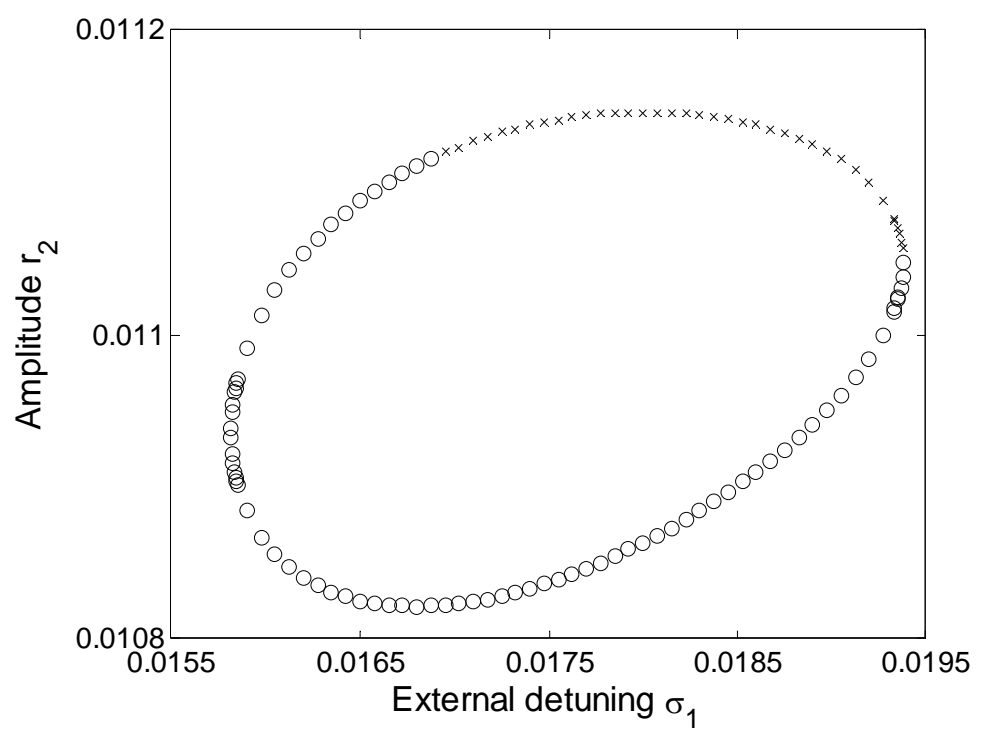

(b) the variation of amplitude $r_{2}$ with external detuning $\sigma_{2}$.

Figure 2. The frequency-response curves for difference resonance Case II for $\alpha_{1}=0.001$, $\alpha_{2}=-0.0053, e_{0}=0.16, \Omega_{0}=1.845$, (a) the variation of amplitude $r_{1}$ with external detuning $\sigma_{2}$, (b) the variation of amplitude $r_{2}$ with external detuning $\sigma_{2}$. Stable solutions are denoted by circles while unstable solutions by crosses. 


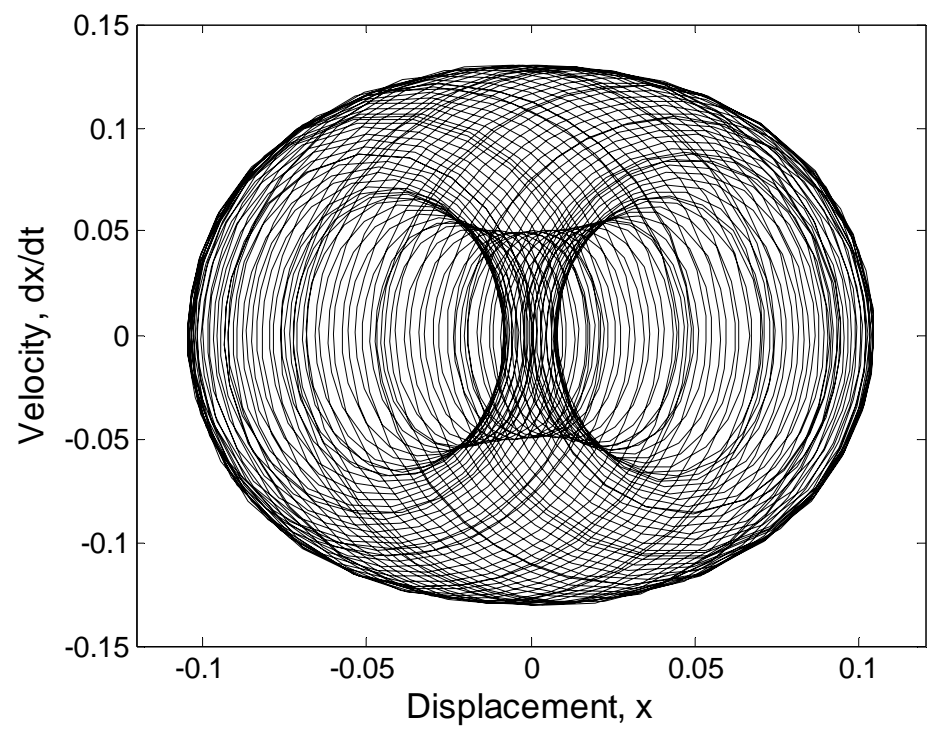

(a) the initial conditions for numerical integration are $(x, \dot{x})=(0.001,0.001)$.

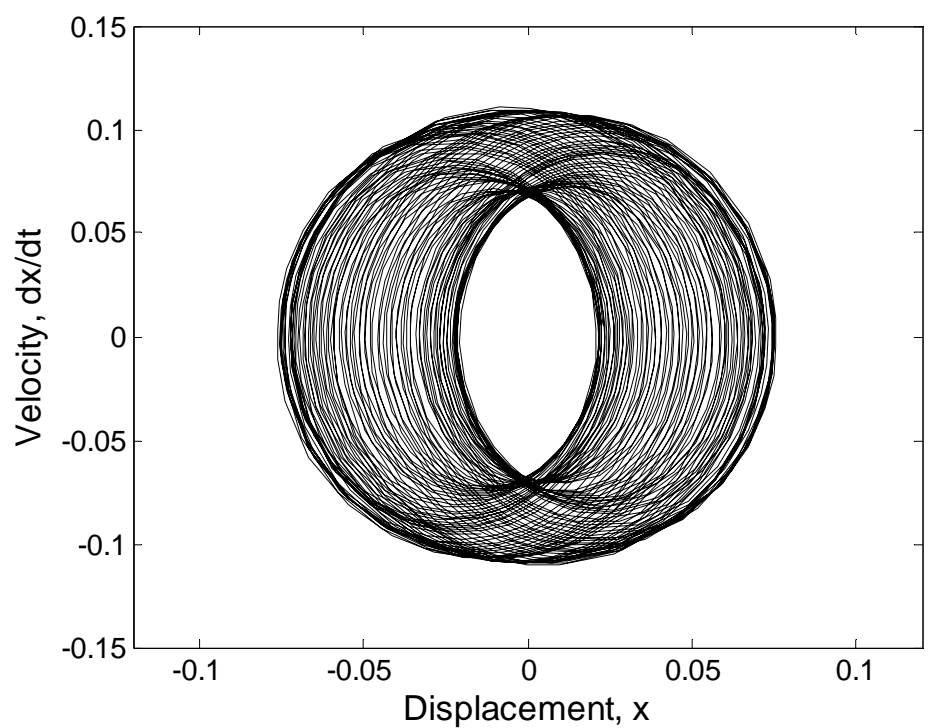

(b) the initial conditions for numerical integration are $(x, \dot{x})=(0.4,0.5)$.

Figure 3. Phase portraits to illustrate the co-existence of two quasi-periodic motions for difference resonance Case II at $\Omega_{0}=1.86094$; (a) the initial conditions for numerical integration are $(x, \dot{x})=(0.001,0.001)$, (b) the initial conditions for numerical integration are $(x, \dot{x})=(0.4,0.5)$. 